\title{
Chromatic Coloring of Distance Graphs I
}

\section{Yegnanarayanan}

Abstract: The primary aim of this paper is to publicize various problems regarding chromatic coloring of finite, simple and undirected graphs. A simple motivation for this work is that the coloring of graphs gives models for a variety of real world problems such as scheduling. We prove some interesting results related to the computation of chromatic number of certain distance graphs and also discuss some open problems.

Keywords: Graph coloring, chromatic number, distance graph.

\section{INTRODUCTION}

A simple graph $\mathrm{G}$ with $(\mathrm{V}, \mathrm{E})$ as (vertex set, edge set) pair is considered as a unit distance graph if $\mathrm{f}: \mathrm{V} \rightarrow \mathrm{R}^{2}$ exists as an embedding with $|f(x)-f(y)|=1$ for each pair $(x, y) \in E$. By $|\cdot|$ we deem an Euclidean norm. Erdos propagated the problem of determination of $\chi\left(G\left(\mathbb{R}^{2},\{1\}\right)\right)$ due to Hadwiger-Nelson. Here $\{1\}$ stands for the distance set $D$ with 1 as only element. So the edge set $E$ of such a graph G evolves when introducing an edge between two elements of $\mathrm{R}^{2}$ separated by a unit distance and $\chi$ stands for the chromatic number. By the chromatic number of a graph we comprehend the least number of colors needed to properly color the vertices of $G$ in such a way that any two vertices that are linked by an edge receive distinct colors. Lately, Grey in [1] found that $\chi\left(G\left(R^{2},\{1\}\right)\right)$ is at least 5 by exhibiting a 5-chromatic graph $\mathrm{G}$ on 1581 vertices of unit distance. It was further improved by Heule in [2] and his unit distance graph $\mathrm{G}\left(\mathbb{R}^{2},\{1\}\right)$ had 553 vertices.

\section{MOTIVATION}

What is it that is common between conducting of examination and organizing sports events? It is clear that both the tasks demand huge planning and scheduling. It is stimulating to note that both the tasks derive a lot of benefits from a portion of graph theory named chromatic coloring.

\section{Scheduling Task}

Suppose that we have a collection of tasks endowed with the counsel regarding which of them cannot be handled simultaneously. The aim is to determine the least time in which all tasks can be completed. We can model this scheduling task with a graph whose vertices denote tasks and the edges among them for conflicts associated with it. That

Manuscript received on June 26, 2021.

Revised Manuscript received on July 01, 2021.

Manuscript published on July 30, 2021.

* Correspondence Author

V. Yegnanarayanan*, Department of Mathematics, Kalasalingam Academy for Research and Education, Deemed to be University, Krishnankoil, Srivilliputhur (Tamil Nadu), India. E-mail: prof.yegna@gmail.com

(c) The Authors. Published by Blue Eyes Intelligence Engineering and Sciences Publication (BEIESP). This is an open access article under the CC BY-NC-ND license (http://creativecommons.org/licenses/by-nc-nd/4.0/) is, an edge is introduced between two vertices iff the respective tasks could not be performed simultaneously. The least time required to complete the task corresponds to the problem of computing the chromatic number of the respective graph. The same idea can be extended to the task of conducting examinations.

In general, it is known that the task of computation of chromatic number unless it is one or two belongs to the class of NP-complete problems. It means no polynomial time step-by-step procedure could find the chromatic number of any given graph.

\section{Mobile / Cellular Networks}

Chromatic coloring could be involved for mobile based communication. GSM is a mobile network where a land area is split into cells (often in the shape of a hexagon) and each cell can be deemed as a transceiver linking the cellular devices within it. To overcome interference, we should avoid using the same channel for two adjacent cells. By the famous 4-color Theorem of graph colorings, we require only 4 distinct channels to assure this constraint independent of the cell shape.

\section{FORBIDDEN GRAPHS}

It has become a practice to call a graph $\mathrm{G}$ as forbidden if it is not a unit distance graph. The problem of deciding whether a given graph is of unit distance belongs to the class of NP-hard problems as it shares the identical complexity with the problem of assessing the validity of sentences within the realm of R's existential theory [3].

In [4] the authors have demonstrated that one of the following two graphs in Fig. 1, namely $G_{1}$, is of unit distance whereas the other, namely $\mathrm{G}_{2}$, is forbidden by introducing the concept of minimality. They called a graph that is forbidden is minimal if every proper subgraph of it is a unit distance graph.

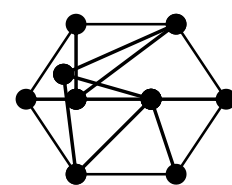

$\mathrm{G}_{1}$

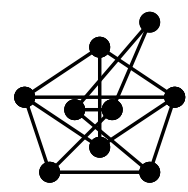

$\mathrm{G}_{2}$
Fig. 1. $G_{1}$ is a Unit Distance $G r a p h$ and $G_{2}$ is a Forbidden Graph

Note that $\mathrm{K}_{4}$, the complete graph on 4 vertices and $\mathrm{K}_{2,3}$, the complete bipartite graph with two vertices in one partite set and three vertices in the other partite set are minimal forbidden graphs. It is widely known that all graphs on and up to less than or equal to five vertices are unit distance graphs provided they do not admit $\mathrm{K}_{4}$ or $\mathrm{K}_{2,3}$ as subgraph.

Published By:

Blue Eyes Intelligence Engineering and Sciences Publication 


\section{Chromatic Coloring of Distance Graphs I}

In [5] the authors showed that the graphs in Figure 2 which constitute a set of graphs that are minimal forbidden on less than equal to 7 vertices. So it reveals that the necessary and sufficient that any graph on and upto 7 vertices is considered as forbidden if it includes a subgraph that is $\cong$ to one of the graphs given in Fig. 2. One can also refer to [6] for more on such forbidden graphs on $\leq 9$ vertices.
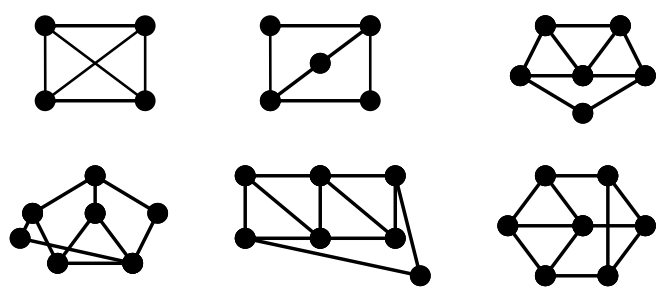

Fig. 2.Set of Graphs that are minimal forbidden on less than or equal to seven vertices

\section{CHROMATIC COLORING OF DISTANCE GRAPHS}

As the problem of computing the exact value of $\chi\left(G\left(\mathbb{R}^{2},\{1\}\right)\right)$ is extremely difficult, researchers considered problems of the next lower levels namely that of finding the exact value of $\chi(G(Z, D))$ where $Z$ has replaced $R^{2}$ and $\{1\}$ is replaced by a well-defined subset of either $Z$ or $N$.

Voigt [7-11] carried out this investigation of these type of integer distance graphs and have achieved several interesting results. Note that any graph $\mathrm{G}$ with finite vertex set can be deemed as a distance graph with integers as its distance set elements. That is, for every such $\mathrm{G}$, one can always find a distance set $D$, and a graph generated with $D$, namely $G_{1}\left(D_{1}\right)$ includes in it an induced subgraph $\mathrm{G}_{2} \cong \mathrm{G}$.

By a k-coloring of a distance graph $G(Z, D)$ we mean a mapping $\mathrm{g}: \mathrm{Z} \rightarrow \mathrm{A}$ where $|\mathrm{A}|=\mathrm{k}$. It is further called a periodic coloring with period $s$, if $g(j+s)=g(j)$ for each $\mathrm{j} \in \mathrm{Z}$. Next, we refer $\mathrm{g}$ as $\mathrm{T}$-congruous if $\mathrm{g}(\mathrm{j}+\mathrm{t}) \neq \mathrm{g}(\mathrm{j})$ for each $j \in Z$ and each $t \in T$. Suppose $T=\{t\}$, we then name such $g$ as t-congruous. Hence, in the case of $g$ being a proper k-coloring of $\mathrm{G}(\mathrm{Z}, \mathrm{D})$ it is evident that it must be D-congruous. Further one can observe that if $g$ is $\mathrm{t}$-congruous periodic with period $\mathrm{s}>\mathrm{t}$ then it is also (ns $\pm \mathrm{t}$ )-congruous for each $n \in \mathbb{N}$.

Proposition 1. If $m \equiv 0(\bmod 3)$ then the chromatic number of the distance graph $G(Z, D)$ with $D=\{1,2, m\}$ is four.

Proof. Assume that $G(Z, D)$ admits a proper three coloring $\mathrm{g}: \mathrm{Z} \rightarrow\{1,2,3\}$. In such a case, g must properly three color a subgraph of $G(Z, D)$. Call such a subgraph as $G\left(Z, D_{1}\right)$ with $D_{1}=\{1,2\}$. Now observe that $g$ is not $t$-congruous if $t \equiv 0$ (mod 3 ). So $g$ is not $\mathrm{m}$-congruous and hence is not a proper three coloring of $\mathrm{G}(\mathrm{Z}, \mathrm{D})$. But then this is a contradiction and so the number of colors required to properly color $G(Z, D)$ is four.

Proposition 2. Let $\mathrm{D}=\left\{\mathrm{u}_{1}, \mathrm{u}_{2}, \mathrm{u}_{3}\right\} \subseteq N$. If $\mathrm{G}(\mathrm{Z}, \mathrm{D})$ permits a proper three coloring $\mathrm{g}: \mathrm{Z} \rightarrow\{1,2,3\}$ and g also properly three colors $\sum_{\mathrm{i}=1}^{3} \mathrm{u}_{\mathrm{i}}$ vertices of $\mathrm{G}(\mathrm{Z}, \mathrm{D})$ that are arranged in increasing order with a distance of separation as unity among any two consecutive vertices then the chromatic number of $\mathrm{G}(\mathrm{Z}, \mathrm{D})$ is at most three.
Proof. Suppose that an arrangement of such a set of $\sum_{\mathrm{i}=1}^{3} \mathrm{u}_{\mathrm{i}}$ vertices is $a, a+1, a+2, \ldots, \mathrm{u}_{1}+\mathrm{u}_{2}+\mathrm{u}_{3}$ and it is properly three colored by g. Let us now attempt to allot a color not used previously to color one after the other the integers left out of $\left\{\mathrm{a}, \mathrm{a}+1, \mathrm{a}+2, \ldots, \sum_{\mathrm{i}=1}^{3} \mathrm{u}_{\mathrm{i}}\right\}$

namely

$\left\{\sum_{\mathrm{i}=1}^{3} \mathrm{u}_{\mathrm{i}}+1, \sum_{\mathrm{i}=1}^{3} \mathrm{u}_{\mathrm{i}}+2, \ldots, 0,-1,-2, \ldots\right\}$. Presume that g cannot have such an extension. Suppose that $\mathrm{w}$ is the least integer such that the colors 1, 2, 3 are assigned to the neighbors of $\mathrm{w}$ previously. Let it by $\mathrm{g}\left(\mathrm{w}-\mathrm{u}_{1}\right)=1, \mathrm{~g}\left(\mathrm{w}-\mathrm{u}_{2}\right)=2, \mathrm{~g}\left(\mathrm{w}-\mathrm{u}_{3}\right)=3$. Then $\mathrm{g}\left(\mathrm{w}-\left(\mathrm{u}_{1}+\mathrm{u}_{2}\right)\right)=3$, $\mathrm{g}\left(\mathrm{w}-\left(\mathrm{u}_{1}+\mathrm{u}_{3}\right)\right)=2$ and $\mathrm{g}\left(\mathrm{w}-\left(\mathrm{u}_{2}+\mathrm{u}_{3}\right)\right)=$ 1. But then this means the existence of a vertex allotted with a color and it is $\mathrm{w}-\left(\sum_{\mathrm{i}=1}^{3} \mathrm{u}_{\mathrm{i}}\right)>1$ and it cannot be assigned with the color 1,2 or 3 . This is against our supposition that $\mathrm{w}$ is least such vertex. Hence the chromatic number of $G(Z, D)$ is at most three.

\section{On r-distance k-coloring}

An r-distance k-coloring of a simple graph $\mathrm{G}$ is a function $\mathrm{f}$ : $\mathrm{V}(\mathrm{G}) \rightarrow\{1,2, \ldots, \mathrm{k}\}$ with the attribute that any two vertices separated by a distance at most $r$ receive different colors. $\chi_{j}(G)$ is the r-distance chromatic number if $k$ is the least integer with respect to which $\mathrm{G}$ has a r-distance k-coloring. If $r=2$, then it is easy to see that $\chi_{2}(G)$ equal to $\chi\left(G^{2}\right)$ where $G^{2}$ is obtained by having $\mathrm{V}\left(\mathrm{G}^{2}\right)$ as $\mathrm{V}(\mathrm{G})$ and $\mathrm{u}_{1} \mathrm{v}_{1} \in \mathrm{E}\left(\mathrm{G}^{2}\right)$ iff $\mathrm{d}\left(\mathrm{u}_{1}\right.$ ,$\left.v_{1}\right)$ is at most 2 . For more on the case when $r=2$ one can see $[12,13]$. A lot of work can be done for other values of $r$.

\section{SOME OPEN PROBLEMS}

Even though a lot of related developments took place while attempting the exact chromatic number computation of $G(R \times R,\{1\})$, not many results were obtained for spaces that are non-euclidean. In [14] the authors have considered the computation of $\chi\left(\mathrm{H}^{2}, \mathrm{D}\right)$ where $\mathrm{H}^{2}$ is the hyperbolic plane. Since $\mathrm{H}^{2}$ includes no homothety, it is worthwhile to probe its $\chi$ value in the case of $\mathrm{D}$ turning out as singleton.

\section{Johnson-Szlam Problem}

Find $\chi(G(Y,\{1\}))$ where $Y$ varies over every possible planar metric spaces.

Deem $Y=(R \times R, d)$ where $d$ is a metric with the translation invariant attribute namely $d(u, v)=d(u+w, v+w)$ for all $u, v, w \in R \times R$. Note that $d$ creates a standard topology on $R \times R$. This implies that every Euclidean disk contains a d disk of identical center. We name such a $d$ as a plane metric and $(R \times R, d)$ a planar metric space. Benoit Kloeckner in [15] demonstrated that 3 is a member of PLANAR, where the set PLANAR implies every possible value of $\chi(G(Y,\{1\}))$ and in [16] the authors established that 4 is a member of PLANAR. One can see [8] for more open problems relatex to Johnson-Szham Problem.

Published By:

Blue Eyes Intelligence Engineering and Sciences Publication

(C) Copyright: All rights reserved. 


\section{ROLE OF SET THEORY AXIOMS IN THE COMPUTATION OF $\chi(\mathrm{G}(\mathrm{R} \times \mathrm{R},\{1\}))$}

In 1951, Erdos and de Bruijn in their seminal paper [17] established that the computation of $\chi(G(R \times R,\{1\}))$ is related to the computation of $\chi$ on some finite subgraph $\mathrm{H} \subseteq \mathrm{G}(\mathrm{R} \times \mathrm{R},\{1\})$. But their proof technique liberally used the axiom of choice. But a related question that tickles every one is what if we do not involve axiom of choice? In [17] the authors have given a distance graph on $\mathrm{R}$ for illustrative purpose, whose $\chi$ computation relies on set theory based system of axioms. They further obtained a conditional result which states: $\chi(G(R \times R,\{1\}))=5,6,7$ in the case of a system that presumes Zermelo-Fraenkel, Dependent Choices and Lebegue Measurability.

\section{AN USEFUL UPPER BOUND FOR CHROMATIC NUMBER}

Proposition 3. Suppose that $G$ is any given graph that is finite, simple and undirected. Then the chromatic number of $\mathrm{G}$ is at most $\left\lceil\left(\frac{\mathrm{t}}{\mathrm{t}+1}\right)(\Delta+2)\right\rceil$.

Proof. Assume that $f_{1} \geq f_{2} \geq \ldots \geq f_{p}$ is the degree based ordering of the vertices of $\mathrm{G}$. Set $\mathrm{s}=(\mathrm{t} / \mathrm{t}+1)(\Delta+2)$, where $\Delta$ stands for the maximum degree among the vertices of $G$, and $t$ is the greatest number of vertices of identical degree with each of them is greater than or equal to $(\Delta+2) / 2$ at least. We assert that $f_{s}$ is strictly not more them $s-1$. As $t$ is at least 1 , the assertion follows when $f_{s}$ is strictly not more than $y-1$ where $y=(\Delta+2) / 2$. Else, we will get that $f_{s}$ is at least $(\Delta+2) / 2$. Note that in the case of $i=1,2, \ldots, s$ we have $f_{i}$ is at most strictly not more than $\Delta-\lceil\mathrm{i} / \mathrm{t}\rceil$ and hence strictly not more than $\Delta-\mathrm{i} / 2+1$. This implies that $\mathrm{f}_{\mathrm{s}}$ is strictly not more than $\Delta-\mathrm{s} / \mathrm{t}+1$ and hence not more than $\mathrm{s}$. So the given graph $\mathrm{G}$ is s-degenerate in the sense that any subgraph of $G$ includes a vertex of degree at most $\mathrm{s}-1$. But then it is already a fact that every s-degenerate graph can be chromatically colored with not more than s colors. So we see that the chromatic number of $G$ is at most $\left\lceil\left(\frac{t}{t+1}\right)(\Delta+2)\right\rceil$.

\section{CONCLUSION}

We have obtained some interesting results concerning the computation of $\chi$ for a certain class of distance graphs and a useful upper bound for $\chi$ of any given graph. We also discussed some open problems and about the practical value of probing $\chi$. The author Yegnanarayanan has also obtained several interesting results on chromatic coloring of graphs.. One can see [18-32].

\section{ACKNOWLEDGMENT}

The author gratefully acknowledges National Board of Higher Mathematics-NBHM, Department of Atomic Energy, Mumbai, India for its financial support vide Ref No. NBHM/RP 8/Feb 2019/Fresh with their letter dated $18^{\text {th }}$ Jan 2021.

\section{REFERENCES}

1. A. D. N. J. de Grey, "The chromatic number of the plane is at least 5," Geombinatorics, vol. 28, no. 1, 2018, pp. 18-31.

2. M. J. H. Henle, "Computing small unit-distance graphs with chromatic number 5," Geoombinatorics, vol. 28, no. 1, 2018, pp. 32-50.

3. M. Schafer, "Realizability of graphs and linkages in Thirty essays on geometric

4. Aiden Globus, and Hans Parshal, "Small unit distance graphs in the plane," Bulletin of the ICA, vol. 90, 2020, pp. 107-138.

5. K. B. Chilakamarri, and C. R. Mahoney, "Maximal and minima forbidden unit-distance graphs in the plane," Bull. Inst. Combin. Appl. vol. 13, 1995, pp. 35-43.

. Aidan Globus, and Hans Parshall, "Small unit-distance graphs in the plane," Bulletin of the Ica, vol. 90, 2020, pp. 107-138. Process. Cybernet. EIK, vol. 28, 1992, pp. 21-28.

8. M. Voigt, "Die chromatische Zahl einer speziellen Klasse unendlicher Graphen," Dissertationsschrifl, Techn. Univ. Ilmenau, 1992.

9. M. Voigt, "Ffirbung yon Distanzgraphen," preprint, 1993. graphs," Discrete App. Math., vol. 51, 1994, pp. 197-209.

11. M. Voigt, and H. Walther, "On the chromatic number of special distance graphs," Discrete Math., vol. 97, 1991, pp. 395-397.

12. F. Kramer, and H. Kramer, "Un problem decoloration des sommets d'un graphe,” C.R. Acad. Sci. Paris A, vol. 268, no. 7, 1969, pp. 46-48.

F. Kramer, and H. Kramer, "A survey on the distance-coloring of

Kahle, "Chromatic number of the hyperplane," Math-overflow,

Kloeckner, "Coloring distance graphs : a few answers and many no. 3, 2015, pp. 117

16. K. B. Chilakamarri, "Unit-distance graphs in Minkowski metric spaces," Geom. Dedicata, vol. 37, no. 3, 1991, pp. 345-356.

17. P. Erdos, and N. G. de Bruijn, "A color problem for infinite graphs and a problem in the theory of relations," Indag. Math., vol. 13, 1951, pp.

V. Yegnanarayanan, Y. Gayathri Narayana and Marius. E. Balas, "On 468; https://doi.org/10.3390/sym10100468 pp 1-13.

19. V. Yegnanarayanan, R. Srikanth, and M. Shanthi, "A brief note on distance graphs,” Asia Life Sciences Supplement, vol. 14, no. 1, 2017, pp. 205-212.

V. Yegnanarayanan, "Chromatic Number of Graphs with Specia no.1, 2014, pp 135-160.

21. V. Yegnanarayanan, and A. Parthiban, "Chromatic Number of Graphs with Special Distance Sets V," Open Journal of Discrete Mathematics, vol. 3, issue 1, 2013, pp. 1-6. doi:10.4236/ojdm.2013.31001 Published ://doi.org/10.4236/ojdm.2013.31001

2. V. Yegnanarayanan, and A. Parthiban., "Vertex Coloring of Certain Distance Graphs," International Journal of Pure and Applied Mathematics, vol. 86, no. 4, 2013, pp. 669-688, htps://doi.org/10.12732/ijpam.v86i4.7

- Yegnanarayanan, "On A Question Concerning Prime Distance Vraphs,” Discrete Mathematics, vol. 245, no.1-3, 2002, pp. 293-298. Mathematika, vol. 3, issue 1, April 2019, pp. 60-69. Available online at www.asiamath.org

25. V. Yegnanarayanan, and A. Parthiban, "Chromatic Number of Graphs with Special Distance Sets III,” J. Math. Comput. Sci., vol. 2, issue 5 2012, pp. 1257-1268.

26. V. Yegnanarayanan, "The chromatic number of generalized Fibonacci prime distance graph,” J. Math. Comput. Sci., vol. 2, issue. 5, 2012, pp. 1451-1463.

V. Yegnanarayanan, "On Prime Distance Graphs and Applications," International Conference on Smart Technologies for Smart Nation (o 2017), to be held at the REVA University, Bangalore, Karnataka, India from 17-19 August 2017, Sponsored by IEEE, Bangalore Section. 
978-1-5386-0569-I\$31.00@2017ＩEEE, Pp 1564-1568, 2017 DOI: $10.1109 /$ SmartTechCon.2017.8358628 Date Added to IEEE Digital Explore 14 $14^{\text {th }}$ May 2018.

28. V. Yegnanarayanan, "On Prime Distance Labelng and applications," International Conference on Smart Technologies for Smart Nation (SmartTechCon 2017), to be held at the REVA University, Bangalore, Karnataka, India from 17-19 August 2017. 978-1-5386-0569-I\$31.00@2017ＩEEE, Pp 1569-1575,2017 DOI: $10.1109 /$ SmartTechCon.2017.8358628Date Added to IEEE Digital Explore $14^{\text {th }}$ May 2018.

29. V. Yegnanarayanan, and A. Parthiban, "On Unit Distance Graphs," Proc of Third International Conference on Discrete Mathematics (ICDM-2013), Karnatak University, Dharwad, June 10-14, 2013, Sponsored by Department of Science and Technology(DST), India and Visvesvaraya Technological University(VTU), Belgaum, India.

30. V. Yegnanarayanan and A. Parthiban, "Chromatic Number of Graphs with Special Distance Sets IV," Proc of International Conference on Applied Mathematics and Theoretical Computer Science, ICAMTCS 2013, Jan 24-25, 2013, pp. 71-76, ISBN 978-93- 82338-30-7 C)2013 Bonfring, Sponsored by IEEE, Madras Section, DRDO, CSIR, DST, organized by St. Xavier's Catholic College of Engineering, Nagercoil, Kanyakumari, India.

31. V. Yegnanarayanan, and A. Parthiban, "Chromatic Number of Certain Graphs," Proc of International Conference on Mathematics in Engineering and Business Management, Stella Maris College, Chennai, vol. I, March 9-11, 2012, pp. 115-118. ISBN 978-81-8286- 015-5.

32. V. Yegnanarayanan, and A. Parthiban, "Chromatic Number of Graphs with Special Distance Sets II", Proc of International Conference on Mathematical Modeling and Applied Soft Computing, CIT, Coimbatore, India, July, 11-13, 2012, pp. 308-3 ISBN No. 978-81- 923752-1-2.

\section{AUTHORS PROFILE}

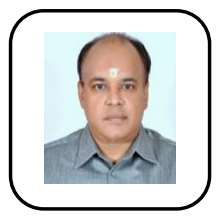

V. Yegnanarayanan, obtained his Full Time $\mathrm{PhD}$ in Mathematics from Annamalai University in Nov 1996. He has over 32 years of teaching and research experience in which 20 years as Professor \& Dean/HOD. He has also worked as a Visiting Scientist on lien in TIFR and IMSC. He has authored 170 research papers and guided 6 students to Ph.D degree . He has delivered a number of invited talks, organized funded conferences, FDP etc, did a lot of review work for MR, zbMATH and reputed journals, completed research projects funded by NBHM-DAE GOI. He has won Sentinel of Science Award from Publons in 2016.

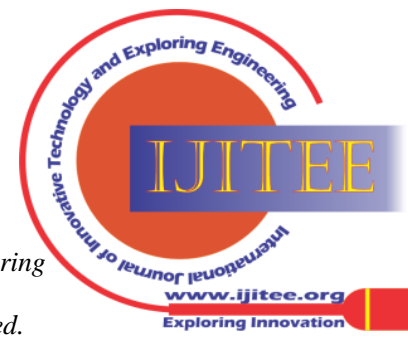

